\title{
Fly walk
}

Two methodological advances enable tracking of the walking behavior of fruit flies at single-leg resolution.

Long gone are the days in which behavior analysis of animal models relied on labor-intensive, frame-byframe manual movie analysis. Newer, sophisticated techniques are becoming available that can automatically annotate complex and detailed behaviors even in tiny animals.

The fruit fly Drosophila melanogaster has been an alltime favorite for studying the genes and neural circuits underlying behavior. Despite its name, the fly is most interesting to researchers when it walks. "To generalize

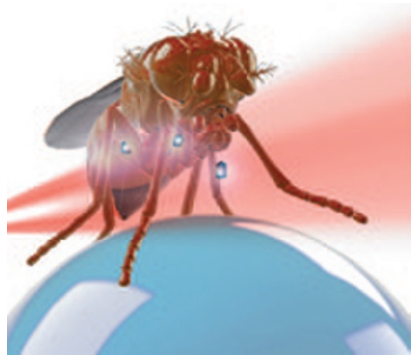

Fly on a floating ball. Image courtesy of B. de Bivort. the behavior very broadly, what flies are doing while they are flying is smelling fruit and going towards the fruit; but once they find it, all the interesting behaviors that flies do-like battling for dominance or mating - are done while they are walking," explains Benjamin de Bivort, who runs a lab as a Junior Fellow at the Rowland Institute at Harvard University.

However, tracking walking behavior in flies has up to now been relegated to relatively lowresolution analyses such as monitoring walking trajectories, the average speed of a group of flies or the ability of a fly to fulfill a simple motor task such as climbing a wall. de Bivort and others soon realized that to understand the mechanisms behind locomotion in this organism, they needed to be able to record the position and motion of each leg. Two methods now provide tools to track and quantify fly walking behavior at this level of detail, expanding the potential of the fruit fly as a model organism for the study of locomotion and the neural circuits that control it.

\section{PROTEOMICS}

\section{Matching marks with mechanisms}

Reagents that recognize specific chemical modifications while ignoring the surrounding protein offer valuable proteomic insights.

Many proteins undergo functional 'fine-tuning' via enzymatic addition and removal of specific chemical groups, a process known as post-translational modification (PTM). Dozens of different PTMs have been identified that affect diverse protein characteristics including localization, stability, activation and so on. By developing 'pan-specific' affinity reagents that recognize target chemical groups in a contextindependent fashion, researchers are now getting a better handle on the distribution and function of poorly understood PTMs at the proteomic scale.

Monoclonal antibodies are still the go-to reagent for such efforts, and phosphospecific antibodies have already generated invaluable insights into serine, threonine and tyrosine phosphorylation. More recently, Tom Muir's team at Princeton University generated a pan-specific antibody against a trickier target: histidine phosphorylation (Kee et al., 2013). This PTM is critical in bacteria and primitive eukaryotes but is difficult to study because of its relative instability; by raising antibodies against a chemical analog of phosphorylated histidine, however, Muir and colleagues have obtained a useful new reagent for proteome-scale analysis of this PTM.

On the other hand, some PTMs have proven intractable to antibody targeting. Histone proteins routinely undergo methylation at lysine residues, modulating the expression levels of nearby genes, and evidence suggests that many nonhistone proteins also undergo lysine methylation. However, the small size of the methyl chemical group has made it difficult to raise broadly PTM-specific antibodies that ignore contributions from nearby amino acids. "We tested a number of commercial antibodies on a peptide array technology we developed so that we could tell how 'pan' something was, and they were terrible," says Or Gozani of Stanford University. 
The first of these methods, developed by Richard Mann and his colleagues at Columbia University, uses an optical technique called frustrated total-internal reflection to track the legs of individual flies as they walk (Mendes et al., 2013). In this setup, the flies walk freely in a small arena with a glass surface. The researchers illuminate the glass, and a camera positioned under the surface detects the interference in the light reflection patterns produced by the insect's legs as it walks. Tracking the fly's footprints is automated thanks to software that the group also developed.

de Bivort, on the other hand, was interested in tracking walking flies over long time periods, and he developed a system in which flies are tethered by their thorax and walk on a treadmill of sorts (Kain et al., 2013). The fly is mounted on top of a clear plastic ball that floats on a cushion of air, and the fly turns the ball as it walks. By making the ball transparent, the researchers managed to image the legs from below using a custom-built imaging system. They glued small pieces of film colored with infrared dye onto each of the fly's legs and illuminated the legs with a laser while recording the fluorescence from below using a couple of cameras. They used another sphere, positioned under the first one, to collimate the image coming from the fly's legs as they moved. de Bivort and colleagues also developed custom software that tracks and quantifies walking behaviors and that uses machine learning approaches to identify instances in which the fly is engaged in specific behaviors such as turning or grooming.

de Bivort now wants to use two-photon microscopy to visualize the activity of specific neurons in the fly's nervous system while tracking walking behavior at high resolution. Pairing detailed behavioral analysis with cellular-level physiological measurements seems a promising path to take.

\section{Erika Pastrana}

\section{RESEARCH PAPERS}

Kain J. et al. Leg-tracking and automated behavioural classification in Drosophila. Nat. Commun. 4, 1910 (2013).

Mendes C.S. et al. Quantification of gait parameters in freely walking wild type and sensory deprived Drosophila melanogaster. Elife 2, e00231 (2013).

As an alternative, Gozani and lab members Kaitlyn Moore and Scott Carlson devised 3xMBT, an affinity reagent engineered from a portion of the human protein L3MBTL1 that contains a trio of methyllysine-binding domains (Moore et al., 2013). "This domain had been characterized as a histone methyl-binding domain, but we knew that it was very nonspecific and only cared about the methylated lysine," says Gozani. Initial experiments with cell lysates verified that 3xMBT could effectively bind a wide range of proteins containing either mono- or dimethylated lysine, with minimal impact from the adjacent primary structure or even other PTMs in nearby positions. This reagent also proved useful for characterizing proteome-wide lysine methylation in a series of mass spectrometry experiments. By using mass spectrometry to compare the cohort of 3xMBT-binding proteins isolated from control cells and those treated with a specific chemical inhibitor of the lysine methyltransferase G9a, the researchers could even scan the proteome for in vivo substrates of this enzyme.

$3 \times$ MBT offers unprecedented access to what now appears to be a fairly widespread PTM, but it still suffers from some important limitations. For example, it is incapable of recognizing trimethylated lysine residues, and some evidence suggests that such PTMs are likely to be especially potent modulators of protein function. Nevertheless, these early results demonstrate that $3 x$ MBT should be sufficient to begin delving into the broad cellular contributions of methyllysine regulation. "I was surprised to find how prevalent it is for pretty much every process - protein translation, splicing, DNA repair, DNA replication and so on," says Gozani. "Altogether there are probably at least 70 candidate lysine methyltransferase enzymes for which we don't know the activity, and we want to characterize them."

\section{Michael Eisenstein}

\section{RESEARCH PAPERS}

Kee, J.-M. et al. A pan-specific antibody for direct detection of protein histidine phosphorylation. Nat. Chem. Biol. doi:10.1038/nchembio.1259 (26 May 2013).

Moore, K.E. et al. A general molecular affinity strategy for global detection and proteomic analysis of lysine methylation. Mol. Cell 50, 444-456 (2013). 